\title{
A NOVEL DUAL-RING MULTI-LAYER CIRCULAR POLARIZED ANTENNA
}

\author{
A.D. Abeygunasekera ${ }^{1}$, C.E. Free ${ }^{1}$, T. Brown ${ }^{2}$ \\ ${ }^{1}$ Advanced Technology Institute, University of Surrey, Guildford, United Kingdom, a.abeygunasekera@ surrey.ac.uk \\ ${ }^{2}$ Centre for Communications Systems Research, University of Surrey, Guildford, United Kingdom
}

Keywords: Circular Polarization, Multilayer Antenna.

\begin{abstract}
A novel circular polarized antenna structure capable of high radiation efficiency, and suitable for use in multilayer microwave circuits is presented. The antenna uses dual ring feed system to enhance radiation. Data is provided for a prototype antenna working at $10 \mathrm{GHz}$.
\end{abstract}

\section{Introduction}

The expanding applications for microwave circuits in current communication systems has generated a need for high density circuit packaging, with integrated antennas. Circuit technologies often need the antenna to be of small scale whilst offering high performance. In this paper, we propose a novel antenna structure capable of high radiation efficiency suitable for use in multilayer microwave circuits. The travelling wave feed system offers high quality circular polarization which is a key requirement for many existing and developing mobile communication systems. The antenna is comprised of two substrate layers with a patch array fed by two circular microstrip channels. The dual ring configuration is used to enhance the radiation of the antenna design. A theoretical investigation based on electromagnetic simulation was performed to validate the new multi-layer antenna at $10 \mathrm{GHz}$.

\section{Antenna Structure and Theory}

The configuration of the antenna is illustrated in figure [1] and is composed of 16 planar rectangular radiating patches. Each patch is linearly polarized and has dimensions $\mathrm{L}_{\mathrm{p}}$ and $\mathrm{W}_{\mathrm{p}}$, where $\mathrm{L}_{\mathrm{p}}$ is the resonant dimension and $\mathrm{W}_{\mathrm{p}}$ is the width of the patch, defining the radiating edges. The patches are excited with a progressive phase lag of $45^{\circ}$ because there is an annular spacing of $\lambda / 8$ between the patches, thus leading to circular polarization. The basic design concept of the antenna was originally established by $\mathrm{K}$. Lum and C. Free [1,2], where a patch array was excited through a circular slot line. The concept of the slotline fed patch was originally established by Tang, et al [3]. The antenna feed was through a circular $50 \Omega$ microstrip channel, of width $\mathrm{W}_{\mathrm{s}}$, which runs on top of the first substrate beneath the patches. The feed is divided into two rings splitting the power from the feed to the patches in the inner and outer circles respectively.

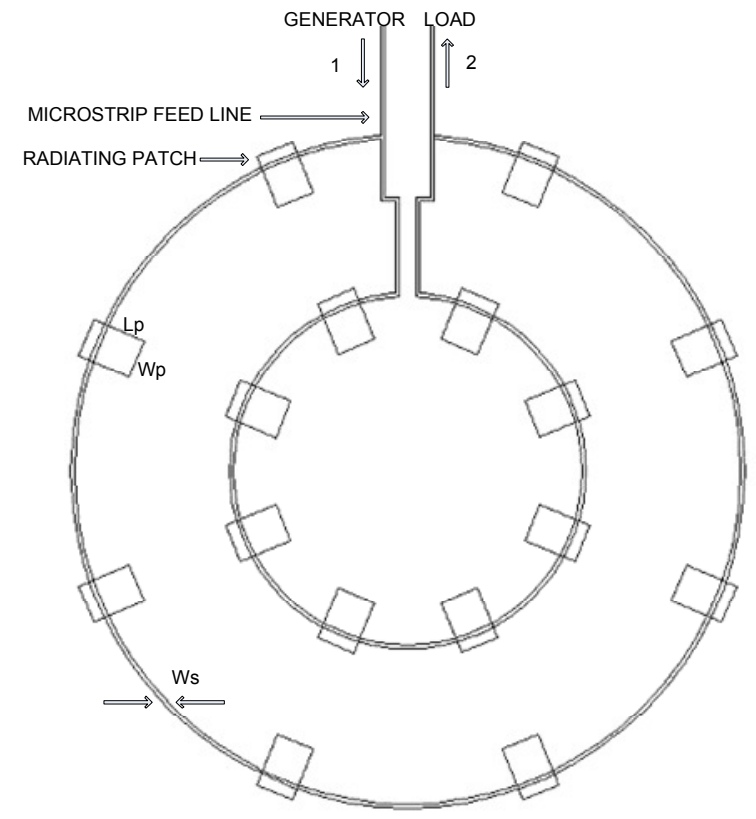

Figure 1. Configuration of the Antenna Structure

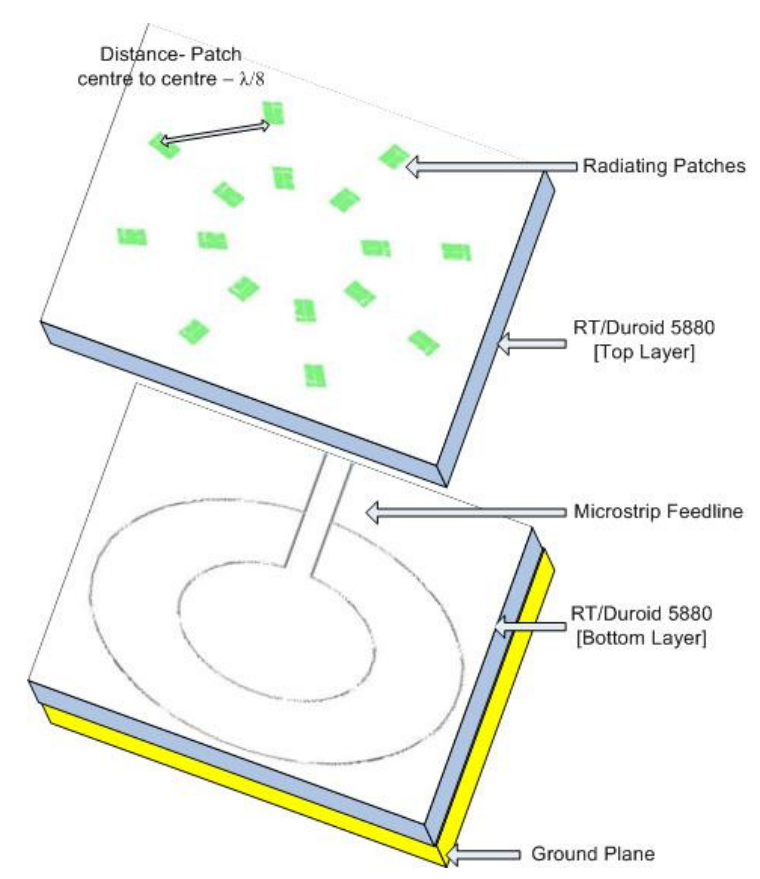

Figure 2. Configuration of the Layers 


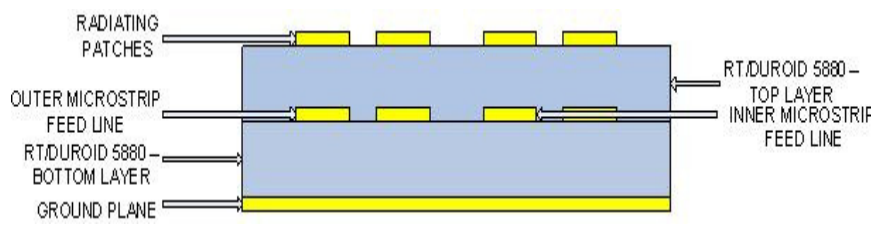

Figure 3. Configuration of the Layers

The test antenna was on two layers of RT/Duroid 5880 having the following parameters: substrate thickness, $\mathrm{h}=0.254 \mathrm{~mm}$; substrate dielectric constant $\varepsilon_{\mathrm{r}}=2.2, ; 0.5-\mathrm{oz}$ electro-deposited copper. The mean diameters of the inner and outer circular microstrip channels were $63.96 \mathrm{~mm}$ and $120.9 \mathrm{~mm}$ respectively, so as to give the correct $\lambda / 8$ spacing between the patches. The key antenna dimensions are:

Length of the patch $\quad L_{p}=9.8 \mathrm{~mm}$

Width of the patch $\quad W_{p}=7 \mathrm{~mm}$

Width of the microstrip line $\mathrm{W}_{\mathrm{s}}=0.747 \mathrm{~mm}$

The overall size of the circuit was $150 \mathrm{~mm} \times 135 \mathrm{~mm}$.

A portion of the signal travelling along the microstrip line will be coupled to the radiating patch through the substrate with the amount of coupling dependent on the relative position of the microstrip line and patch. Because the feeding signals are not terminated at the radiating patch like other feeding techniques, the continuous traveling wave is then used to excite subsequent radiating patches to form a travelling wave antenna structure.

\section{Results}

The structure was modelled using an electromagnetic simulator Momentum (AGILENT Advanced Design System).

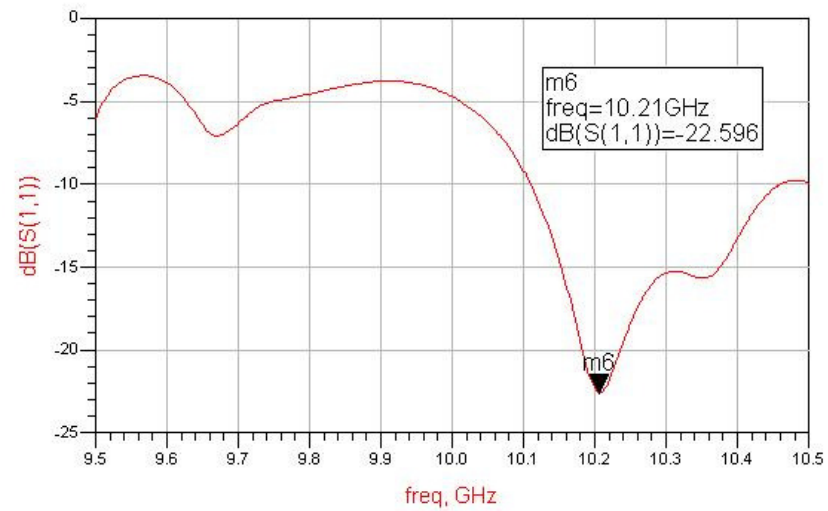

Figure 4. S-Parameters of the Antenna - S11

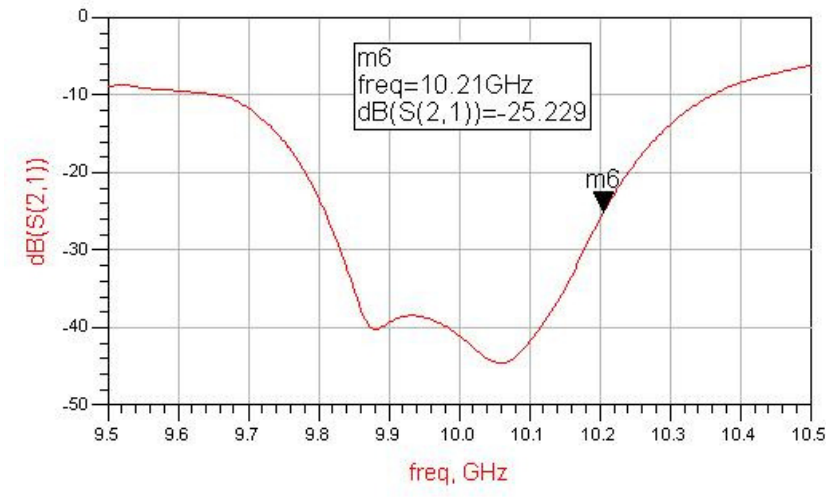

Figure 5. S-Parameters of the Antenna - S21

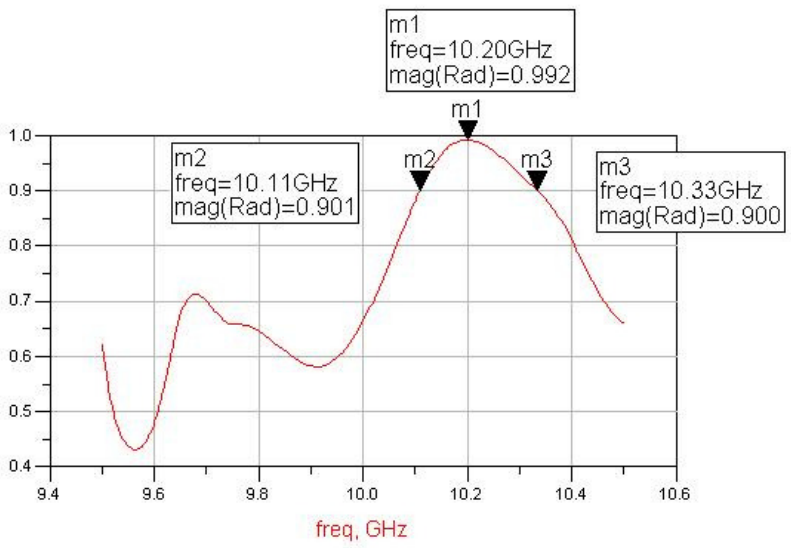

Figure 6. Relative Power Radiated from the Antenna

The optimum frequency of operation is $10.21 \mathrm{GHz}$. This is slightly different from the designed value of $10 \mathrm{GHz}$ because no matching was included to account for the reactance presented by the patches to the feed line. As seen from Fig. 3, the return loss of the antenna is $-22.596 \mathrm{~dB}$ at $10.21 \mathrm{GHz}$. In this antenna structure the travelling wave can be reflected, radiated, or dissipated in the substrate. The amount of signal dissipated in the substrate is insignificant. The reflection as seen from fig 3. is negligible. Therefore most of the signal traveling through the feed has been radiated. S21 is calculated to be $-25.229 \mathrm{~dB}$ for the above design. This indicates radiation loss between the input port and the output port.

Furthermore, from fig 4 . the radiation from the antenna design was calculated to be $99.2 \%$ of the power at the optimum frequency of operation, $10.2 \mathrm{GHz}$. The bandwidth of the antenna is $220 \mathrm{MHz}$. Therefore the design is considered to be useful for narrowband applications.

\section{Conclusion}

The concept of novel circular polarized patch antenna has been established. In this paper a radiation enhancement method using a dual ring feed system was demonstrated. This antenna is particularly suitable for inclusion in highly integrated multilayer transceivers. 


\section{References}

[1] K.M. Lum; T. Tick; C. Free; H. Jantunen, "Design and Measurement Data for a Microwave Dual-CP Antenna Using a New Travelling-Wave Feed Concept", IEEE Trans. On Microwave Theory and Techniques, Vol. 54, no. 6, pp. 2880-2886, June 2006.

[2] K. M. Lum, C. Laohapensaeng, and C. E. Free, "A novel traveling-wave feed technique for circularly polarized planar antennas," IEEE Microwave Wireless Components Letters, vol. 15, no. 3, pp. 180-182, March 2005

[3] K. P. Tang, C. E. Free, and G. F. Goldspink, "A novel slotline-fed microstrin patch antenna", IEEE Antennas and Propagation Conf:, vol. 1, no. 407, pp. 235-238, April 1995. 